Vol. 50 (1994) [5-15]

\title{
A NOTE ON GRAPHS WITH A PRESCRIBED ADJACENCY PROPERTY
}

\author{
W. Ananchuen and L. Caccetta
}

\begin{abstract}
Let $m$ and $n$ be nonnegative integers and $k$ be a positive integer. A graph $G$ is said to have property $P(m, n, k)$ if for any set of $m+n$ distinct vertices of $G$ there are at least $k$ other vertices, each of which is adjacent to the first $m$ vertices of the set but not adjacent to any of the latter $n$ vertices. The problem that arises is that of characterising graphs having property $P(m, n, k)$. This problem has been considered by several authors and a number of results have been obtained. In this paper, we establish a lower bound on the order of a graph having property $P(m, n, k)$. Further, we show that all sufficiently large Paley graphs satisfy properties $P(1, n, k)$ and $P(n, 1, k)$.
\end{abstract}

\section{INTRODUCTION}

For our purposes graphs are finite, loopless and have no multiple edges. For the most part our notation and terminology follows that of Bondy and Murty [7]. Thus $G$ is a graph with vertex set $V(G)$, edge set $E(G), \nu(G)$ vertices, $E(G)$ edges, minimum degree $\delta(G)$ and maximum degree $\Delta(G)$. However, we denote the complement of $G$ by $\bar{G}$.

In the application of graph theory to problems arising in network design and analysis, the requirements of the network (such as efficiency and reliability) can often be translated into adjacency restrictions on the graph representing the network. Here we consider graphs having a prescribed adjacency property.

More specifically, a graph $G$ is said to have property $P(m, n, k)$ if for any set of $m+n$ distinct vertices there are at least $k$ other vertices, each of which is adjacent to the first $m$ vertices but not adjacent to any of the latter $n$ vertices. The class of graphs having property $P(m, n, k)$ is denoted by $\mathcal{G}(m, n, k)$. Observe that if $G \in \mathcal{G}(m, n, k)$, then $\bar{G} \in \mathcal{G}(n, m, k)$. The cycle $C_{\nu}$ of length $\nu$ is a member of $\mathcal{G}(1,1,1)$ for every $\nu \geqslant 5$. The well-known Petersen graph is a member of $\mathcal{G}(1,2,1)$ and also of $\mathcal{G}(1,1,2)$. Despite these relatively simple examples, few members of $\mathcal{G}(m, n, k)$ have been found. Recently [3] we constructed several classes of graphs in $\mathcal{G}(1, n, k)$.

Received 22 January 1994

This work has been supported by an Australian Research Council Grant A48932119.

Copyright Clearance Centre, Inc. Serial-fee code: 0004-9729/94 \$A2.00+0.00. 
The problem that arises is that of characterising the class $\mathcal{G}(m, n, k)$. Two particularly interesting problems that arise concern the functions

$$
p(m, n, k)=\min \{\nu(G): G \in \mathcal{G}(m, n, k)\}
$$

and

$$
q(\ell,(m, n, k))=\min \{\varepsilon(G): \nu(G)=\ell \text { and } G \in \mathcal{G}(m, n, k)\}
$$

The only result concerning the latter function is due to Erdös and Moser [8] who determined $q(\ell,(m, 0,1))$. Exoo [9], established bounds on $p(n, n, 1)$. Blass and Harary [5] established, using probabilistic methods, that almost all graphs have property $P(n, n, 1)$. From this it is not too difficult to show that almost all graphs have property $P(m, n, k)$. Despite this result, few graphs have been constructed which exhibit the property $P(m, n, k)$.

Properties of graphs in $\mathcal{G}(m, n, k)$ were given in $[1,2,9]$. In particular, in [2] we proved that

$$
p(2,2, k) \geqslant \begin{cases}34, & \text { for } k=1, \\ 8 k+25, & \text { for odd } k \geqslant 3, \\ 8 k+21, & \text { otherwise. }\end{cases}
$$

In Section 2, we prove that

$$
p(n, n, k) \geqslant 4^{n-1}\left[2(n+k)+\frac{1}{2}\left(3+(-1)^{n+k+1}\right)+\frac{1}{3}\right]-\frac{1}{3} .
$$

This generalises a result of Exoo [9].

An important graph in the study of the class $\mathcal{G}(m, n, k)$ is the so-called Paley graph $G_{q}$ defined as follows. Let $q \equiv 1(\bmod 4)$ be a prime power. The vertices of $G_{q}$ are the elements of the finite field (Galois field) $\mathbb{F}_{q}$. Two vertices $a$ and $b$ are joined by an edge if and only if their difference is a quadratic residue, that is $a-b=y^{2}$ for some $y \in \mathbb{F}_{q}$.

For a prime $p \equiv 1(\bmod 4)$, Blass, Exoo and Harary [4] showed that $G_{p} \in$ $\mathcal{G}(n, n, 1)$ for $p>n^{2} 2^{4 n}$. In [2] we improved this result by showing that for a prime power $q \equiv 1(\bmod 4), G_{q} \in \mathcal{G}(n, n, k)$ for every $q>\left\{(2 n-3) 2^{2 n-1}+2\right\} \sqrt{q}+$ $(n+2 k-1) 2^{2 n-1}-2 n^{2}-1$. Further, we proved that $G_{q} \in \mathcal{G}(m, n, k)$ for every $q>\left\{(t-3) 2^{t-1}+2\right\} \sqrt{q}+(t+2 k-1) 2^{t-1}-1$, where $t \geqslant m+n ;$ and $G_{q} \in \mathcal{G}(1,2, k)$ for every $q>(1+2 \sqrt{2 k})^{2}$. Computational results were also presented to establish the smallest Paley graphs in $\mathcal{G}(2,2, k)$ for small $k$.

In Section 3, we prove that $G_{q} \in \mathcal{G}(1, n, k) \cap \mathcal{G}(n, 1, k)$ for every $q>\left\{(n-2) 2^{n}+\right.$ $2\} \sqrt{q}+(n+2 k-1) 2^{n}-2 n-1$. Computational results show that this result is best possible for $n=1$ (all $k$ ) and for $n=2$ (most $k$ ), and close to best possible for $n=3$. 


\section{LOWER BOUND ON $p(m, n, k)$}

We begin with some notation and terminology. For disjoint subsets $A$ and $B$ of $V(G)$ we denote by $N(A / B)$ the set of vertices of $G$ not in $A \cup B$ which are adjacent to each vertex of $A$ and not adjacent to any vertex of $B$. When $A=\left\{a_{1}, a_{2}, \ldots, a_{m}\right\}$ and $B=\left\{b_{1}, b_{2}, \ldots, b_{n}\right\}$ we write for convenience $N(A / B)$ as $N\left(a_{1}, a_{2}, \ldots, a_{m} / b_{1}, b_{2}, \ldots, b_{n}\right)$. Where appropriate, lower case letters will denote the cardinality of the set defined by the corresponding upper case letters. Thus for example, $n(a / b)=|N(a / b)|$. Finally, for $X \subseteq V(G)$ we let $G[X]$ denote the subgraph of $G$ induced by $X$,

An $r$-regular graph of order $\nu$ is called strongly regular with parameters $(\nu, r, \lambda, \mu)$ if $G$ has the property that any two adjacent vertices have exactly $\lambda$ common neighbours and any two non-adjacent vertices have exactly $\mu$ common neighbours.

We make use of the following results:

Lemma 2.1. (Exoo [9]). If $G \in \mathcal{G}(1,1, k)$, then $\nu(G) \geqslant 4 k+1$, with equality holding if and only if there exists a strongly regular graph with parameters $(4 k+1,2 k, k-1, k)$.

Lemma 2.2. (Ananchuen and Caccetta [1]). For $1 \leqslant j \leqslant n, \mathcal{G}(m, n, k) \subseteq$ $\mathcal{G}(m, n-j, k+j)$.

The following is a useful lemma in our work.

LEMMA 2.3. Let $G \in \mathcal{G}(n, n, k)$ and $\left\{u_{1}, u_{2}, \ldots, u_{n-1}, v_{1}, v_{2}, \ldots, v_{n-1}\right\}$ be a set of $2 n-2$ vertices of $G$. Then the subgraph

$$
H=G\left[N\left(u_{1}, u_{2}, \ldots, u_{n-1} / v_{1}, v_{2}, \ldots, v_{n-1}\right)\right]
$$

has $\delta(H) \geqslant n+k$.

Proof: Suppose to the contrary that $d_{H}(x)=d \leqslant n+k-1$. Let $y_{1}, y_{2}, \ldots, y_{d}$ be the neighbours of $x$ in $H$. First we prove that $d \geqslant n$. Suppose $d<n$. Since $G \in \mathcal{G}(n, n, k)$, there exists $w \in N\left(y_{1}, y_{2}, \ldots, y_{d} / u_{1}, u_{2}, \ldots, u_{n-1}\right)$ and a $y \in$ $N\left(u_{1}, u_{2}, \ldots, u_{n-1}, x / v_{1}, v_{2}, \ldots, v_{n-1}, w\right)$. Thus $y \in V(H)$ and, since $y$ is adjacent to $x, y=y_{i}$ for some $i$. Hence, $y$ is adjacent to $w$, a contradiction. Therefore $d \geqslant n$ as required. Now consider a vertex $z \in N\left(y_{1}, y_{2}, \ldots, y_{n} / u_{1}, u_{2}, \ldots, u_{n-1}, x\right)$; such a $z$ exists since $G \in \mathcal{G}(n, n, k)$. Clearly $n\left(u_{1}, u_{2}, \ldots, u_{n-1}, x / v_{1}, v_{2}, \ldots, v_{n-1}, z\right) \leqslant$ $k-1$, a contradiction. This proves that $\delta(H) \geqslant n+k$.

We are now ready to prove the main result in this section.

THEOREM 2.1.

$$
p(n, n, k) \geqslant 4^{n-1}\left[2(n+k)+\frac{1}{2}\left\{3+(-1)^{n+k+1}\right\}+\frac{1}{3}\right]-\frac{1}{3} .
$$


Proof: Let $G \in \mathcal{G}(n, n, k)$. For $1 \leqslant i \leqslant n$ define

$$
\mathcal{I}_{i}=\{A: A \subseteq V(G) \text { and }|A|=i\} .
$$

Clearly for disjoint sets $A$ and $B$ in $\mathcal{I}_{i}, N(A / B) \neq \emptyset$. Thus the subgraph $B=$ $G[N(A / B)]$ has vertices; in fact $\nu(H) \geqslant k$. For $1 \leqslant i \leqslant n$, let

$$
h_{i}^{*}=\min \left\{n(A / B): A \cap B=\emptyset, A, B \in \mathcal{I}_{i}\right\} .
$$

Suppose that $h_{i}^{*}=n\left(A^{*} / B^{*}\right)$ and let

$$
\boldsymbol{H}_{i}^{*}=G\left[N\left(A^{*} / B^{*}\right)\right]
$$

Observe that $G \in \mathcal{G}\left(1,1, h_{1}^{*}\right)$. We show that $B_{i}^{*} \in \mathcal{G}\left(1,1, h_{i+1}^{*}\right)$ for each $1 \leqslant i \leqslant$ $n-2$.

Consider the graph $H_{i}^{*}=G\left[N\left(A^{*} / B^{*}\right)\right]$. Then for any $a, b \in V\left(B_{i}^{*}\right)$, the number $n^{*}(a / b)$ of vertices of $H_{i}^{*}$ joined to $a$ but not joined to $b$ satisfies

$$
\begin{aligned}
n^{*}(a / b) & =n\left(A^{*} \cup\{a\} / B^{*} \cup\{b\}\right) \\
& \geqslant h_{i+1}^{*} .
\end{aligned}
$$

Thus, as $a$ and $b$ are arbitrary, $H_{i}^{*} \in \mathcal{G}\left(1,1, h_{i+1}^{*}\right)$. Now, by Lemma 2.1,

$$
h_{i}^{*} \geqslant 4 h_{i+1}^{*}+1, \quad 1 \leqslant i \leqslant n-2 .
$$

Consequently, since $G \in \mathcal{G}\left(1,1, h_{i}^{*}\right)$, we have

$$
\begin{aligned}
\nu(G) & \geqslant 4 h_{1}^{*}+1 \\
& \geqslant 4\left(4 h_{2}^{*}+1\right)+1 \\
& \geqslant 4\left(4\left(4 h_{3}^{*}+1\right)+1\right)+1 \\
& \vdots \\
& \geqslant 4^{n-1} h_{n-1}^{*}+\frac{4^{n-1}-1}{3} .
\end{aligned}
$$

We next prove that

$$
h_{n-1}^{*} \geqslant 2(n+k)+\frac{1}{2}\left[3+(-1)^{n+k+1}\right] .
$$

By Lemma 2.3, $\delta\left(H_{n-1}^{*}\right) \geqslant n+k$. Further, since $\bar{G} \in \mathcal{G}(n, n, k)$ we also have $\delta\left(\bar{H}_{n-1}^{*}\right) \geqslant n+k$. Consequently, $h_{n-1}^{*} \geqslant 2(n+k)+1$, proving (2.3) when $n+k$ 
is even. When $n+k$ is odd, then at least one of $H_{n-1}^{*}$ or $\bar{H}_{n-1}^{*}$ contains a vertex of degree at least $n+k+1$ and thus (2.3) also holds. Now (2.2) and (2.3) together yield (2.1). This completes the proof of the theorem.

Corollary. Let $t=\min \{m, n\}$ and $k^{\prime}=k+|m-n|$. Then

$$
p(m, n, k) \geqslant 4^{t-1}\left[2\left(t+k^{\prime}\right)+\frac{1}{2}\left\{3+(-1)^{t+k^{\prime}+1}\right\}+\frac{1}{3}\right]-\frac{1}{3} .
$$

Proof: Since $G \in \mathcal{G}(m, n, k)$ implies that $\bar{G} \in \mathcal{G}(n, m, k)$, we have $p(m, n, k)=$ $p(n, m, k)$. Hence

$$
\begin{aligned}
p(n, m, k) & =p(t, t+|m-n|, k) \\
& \geqslant p\left(t, t, k^{\prime}\right) \quad(\text { by Lemma 2.2) } \\
& \geqslant 4^{t-1}\left[2\left(t+k^{\prime}\right)+\frac{1}{2}\left\{3+(-1)^{t+k^{\prime}+1}\right\}+\frac{1}{3}\right]-\frac{1}{3}
\end{aligned}
$$

as required.

REMARK. A consequence of the proof of Theorem 2.1 is that $\mathcal{G}(n, n, k) \subseteq \mathcal{G}(n-1$, $\left.n-1, h_{n-1}^{*}\right)$. For the particular case $n=2, k=1$, we have $\mathcal{G}(2,2,1) \subseteq \mathcal{G}(1,1,8)$. Thus, by Lemma $2.1, p(2,2,1) \geqslant 33$ with equality possible only if there exists a strongly regular graph with parameters $(33,16,7,8)$. It is well-known that such a graph does not exist. Hence, $p(2,2,1) \geqslant 34$. To date, the smallest graph in $\mathcal{G}(2,2,1)$ constructed is the Paley graph on 61 vertices.

\section{Paley graphs}

The Paley graph $G_{q}$ of order $q \equiv 1(\bmod 4), q$ a prime power, was defined in the introduction. Observe that $G_{q}$ is self-complementary. Further, see [6], it is strongly regular with parameters $(4 t+1,2 t, t-1, t)$ when $q=4 t+1$. In [2] we proved that $G_{q} \in \mathcal{G}(m, n, k)$ for all $q>\left\{(t-3) 2^{t-1}+2\right\} \sqrt{q}+(t+2 k-1) 2^{t-1}-1$, where $t$ is an integer satisfying $t \geqslant m+n$. In this section we prove a sharper result for the case when one of $m$ or $n$ is 1 .

We make use of the following basic notation and terminology. Let $\mathbb{F}_{q}$ be a finite field of order $q$, where $q$ is a prime power.

A character $\chi$ on $\mathbb{F}_{q}^{*}$, the multiplicative group of the non-zero elements of $\mathbb{F}_{q}$, is a map from $\mathbb{F}_{q}^{*}$ to the multiplicative group of complex numbers with $|\chi(x)|=1$ for all $x$ and with

$$
\chi(x y)=\chi(x) \chi(y)
$$

for any $x, y \in F_{q}^{*}$. Since $\chi(1)=\chi(1) \chi(1)$, we have $\chi(1)=1$. 
Among the characters of $\mathbb{F}_{q}^{*}$ we have the principal character $\chi_{0}$ defined by $\chi_{0}(x)=$ 1 for all $x \in \mathbb{F}_{q}^{*}$; all other characters of $\mathbb{F}_{q}^{*}$ are called non-principal.

It will be convenient to extend the definition of non-principal character $\chi$ to the whole $\mathbb{F}_{q}$ by putting $\chi(0)=0$.

If $\chi$ is a non-principal character on $\mathbb{F}_{q}$, it is well-known (see [10]) that

$$
\sum_{x \in T_{q}} \chi(x)=0
$$

It follows that, for $a \in \mathbb{F}_{q}$

$$
\sum_{x \in \mathbb{F}_{q}} \chi(x-a)=0
$$

The following lemma, due to Schmidt [11]; is very useful in our work.

LEMmA 3.1. Let $\chi$ be a non-principal character on $\mathbb{F}_{q}$. If $a_{1}, a_{2}, \ldots, a_{s}$ are distinct elements of $\mathbb{F}_{q}$, then

$$
\left|\sum_{x \in \mathbb{F}_{q}} \chi\left\{\left(x-a_{1}\right)\left(x-a_{2}\right) \ldots\left(x-a_{s}\right)\right\}\right| \leqslant(s-1) \sqrt{q}
$$

Let $q$ be a power of an odd prime. We define a quadratic (residue) character $\eta$ on $\mathbb{F}_{q}$ by

$$
\eta(a)=a^{(q-1) / 2}, \quad \text { for all } a \in \mathbb{F}_{q}
$$

Equivalently, $\eta$ is 1 on squares, 0 at 0 , and -1 otherwise. Therefore, $\eta$ is a nonprincipal character.

The following lemma was proved in [2].

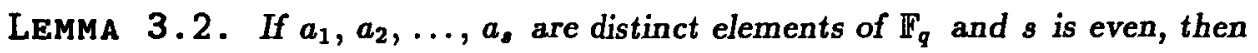

$$
\sum_{\boldsymbol{x} \in \mathbf{F}_{q}} \eta\left\{\left(x-a_{1}\right)\left(x-a_{2}\right) \ldots\left(x-a_{s}\right)\right\}=-1 \pm \sum_{x \in \mathbf{F}_{q}} \eta\left\{\left(x+b_{1}\right)\left(x+b_{2}\right) \ldots\left(x+b_{s-1}\right)\right\}
$$

for some distinct elements $b_{1}, b_{2}, \ldots, b_{s-1}$ of $\mathbb{F}_{q}$.

Using (3.2) and Lemma 3.1 we have the following corollaries to Lemma 3.2.

Corollary 1. For $a, b \in \mathbb{F}_{q}$ with $a \neq b$

$$
\sum_{x \in \mathbf{F}_{q}} \eta\{(x-a)(x-b)\}=-1
$$


CoRollary 2. Let $a_{1}, a_{2}, \ldots, a$, be distinct elements of $\mathbb{F}_{q}$. Then for even $s$

$$
\left|\sum_{x \in \mathbf{F}_{q}} \eta\left\{\left(x-a_{1}\right)\left(x-a_{2}\right) \ldots\left(x-a_{s}\right)\right\}\right| \leqslant 1+(s-2) \sqrt{q} .
$$

In addition to the above, we need the following lemma.

LEMмA 3.3. Let $a \in V\left(G_{q}\right)$ and $B=\left\{b_{1}, b_{2}, \ldots, b_{n}\right\}$ a subset of $n$ vertices of G-a.Put

$$
g=\sum_{x \in \mathbb{F}_{q}}\{1+\eta(x-a)\} \prod_{i=1}^{n}\left\{1-\eta\left(x-b_{i}\right)\right\} .
$$

As usual, an empty product is defined to be 1 . Then

$$
g \geqslant q-\left\{(n-2) 2^{n}+2\right\} \sqrt{q}-\left\{2^{n}-2 n-1\right\}
$$

Proof: We can write

$$
\begin{aligned}
g= & \sum_{x \in \mathbb{F}_{q}} 1+\sum_{x \in \mathbb{F}_{q}}\left\{\eta(x-a)-\sum_{i=1}^{n} \eta\left(x-b_{i}\right)\right\} \\
& +\sum_{x \in \mathbb{F}_{q}}\left\{\sum_{i=1}^{n-1} \sum_{j=i+1}^{n} \eta\left\{\left(x-b_{i}\right)\left(x-b_{j}\right)\right\}-\sum_{i=1}^{n} \eta\left\{(x-a)\left(x-b_{i}\right)\right\}\right\} \\
& +\ldots+\sum_{x \in \mathbb{F}_{q}} \prod_{i=1}^{n} \eta\left\{(x-a)\left(b-b_{i}\right)\right\} .
\end{aligned}
$$

Observe that the first term of the expression is equal to $q$ and from (3.2) the second term is 0 . Using Corollary 1 of Lemma 3.2 the third term of the above expansion is equal to $n-\left(\begin{array}{c}n \\ 2\end{array}\right)=\left(3 n-n^{2}\right) / 2$. Hence,

$$
\begin{aligned}
\mid g-q & -\frac{1}{2}\left(3 n-n^{2}\right)|\leqslant| \sum_{z \in E_{q}} \sum_{i=1}^{n-1} \sum_{j=i+1}^{n} \sum_{k=j+1}^{n+1} \eta\left\{\left(x-c_{i}\right)\left(x-c_{j}\right)\left(x-c_{k}\right)\right\} \mid \\
+ & \left|\sum_{x \in \mathbf{F}_{q}} \sum_{i=1}^{n-2} \sum_{j=i+1}^{n-1} \sum_{k=j+1}^{n} \sum_{\ell=k+1}^{n+1} \eta\left\{\left(x-c_{i}\right)\left(x-c_{j}\right)\left(x-c_{k}\right)\left(x-c_{\ell}\right)\right\}\right|
\end{aligned}
$$

$$
+\ldots+\left|\sum_{x \in \mathbf{F}_{q}} \prod_{i=1}^{n+1} \eta\left(x-c_{i}\right)\right|
$$


where $\left\{c_{1}, c_{2}, \ldots, c_{n+1}\right\}=B \cup\{a\}$.

Now Lemma 3.1 and Corollary 2 of Lemma 3.2 together imply that

$$
\begin{aligned}
& \left|\sum_{x \in F_{q}} \sum_{i_{1}<i_{2}<\ldots<i_{1}} \eta\left\{\left(x-c_{i_{1}}\right)\left(x-c_{i_{2}}\right) \ldots\left(x-c_{i_{s}}\right)\right\}\right| \\
& \leqslant \begin{cases}\left(\begin{array}{c}
n+1 \\
s
\end{array}\right)(s-1) \sqrt{q}, & \text { if } s \text { is odd } \\
\left(\begin{array}{c}
n+1 \\
s
\end{array}\right)\{1+(s-2) \sqrt{q}\}, & \text { if } s \text { is even. }\end{cases}
\end{aligned}
$$

Making use of (3.4) we get from (3.3)

(3.5)

$$
\begin{aligned}
\mid g-q & -\frac{1}{2}\left(3 n-n^{2}\right) \mid \leqslant \sum_{\substack{s=3 \\
s \text { odd }}}^{n+1}\left(\begin{array}{c}
n+1 \\
s
\end{array}\right)(s-1) \sqrt{q}+\sum_{\substack{s=4 \\
\text { seven }}}^{n+1}\left(\begin{array}{c}
n+1 \\
s
\end{array}\right)\{1+(s-2) \sqrt{q}\} \\
& =\left\{\sum_{s=3}^{n+1}\left(\begin{array}{c}
n+1 \\
s
\end{array}\right) s-\sum_{s=3}^{n+1}\left(\begin{array}{c}
n+1 \\
s
\end{array}\right)-\sum_{\substack{s=4 \\
\text { seven }}}^{n+1}\left(\begin{array}{c}
n+1 \\
s
\end{array}\right)\right\} \sqrt{q}+\sum_{\substack{s=4 \\
\text { seven }}}^{n+1}\left(\begin{array}{c}
n+1 \\
s
\end{array}\right) \\
& =\left\{(n-2) 2^{n}+2\right\} \sqrt{q}+2^{n}-\frac{1}{2}\left(n^{2}+n\right)-1 .
\end{aligned}
$$

Hence

$$
\begin{aligned}
g & \geqslant q+\frac{1}{2}\left(3 n-n^{2}\right)-\left\{(n-2) s^{n}+2\right\} \sqrt{q}-\left\{2^{n}-\frac{1}{2}\left(n^{2}+n\right)-1\right\} \\
& =q-\left\{(n-2) 2^{n}+2\right\} \sqrt{q}-\left\{2^{n}-2 n-1\right\}
\end{aligned}
$$

as required.

Before stating and proving our main result of this section we make the following observation. If $a$ and $b$ are any vertices of $G_{q}, q \equiv 1(\bmod 4)$ a prime power, then

$$
\eta(a-b)= \begin{cases}1, & \text { if } a \text { adjacent to } b \\ 0, & \text { if } a=b \\ -1, & \text { otherwise. }\end{cases}
$$

Further, $\eta(-a)=\eta(a)$ for any $a \in \mathbb{F}_{q}$.

Theorem 3.1 . Let $q \equiv 1(\bmod 4)$ be a prime power and $k$ be a positive integer. If

$$
g>\left\{(n-2) 2^{n}+2\right\} \sqrt{q}+(n+2 k-1) 2^{n}-2 n-1,
$$


then $G_{q} \in \mathcal{G}(1, n, k) \cap \mathcal{G}(n, 1, k)$.

ProOF: Since $G_{q}$ is a self-complementary graph, it is sufficient to prove that $G_{q} \in \mathcal{G}(1, n, k)$. Let $a$ be any vertex of $G_{q}$ and $B=\left\{b_{1}, b_{2}, \ldots, b_{n}\right\}$ a set of $n$ vertices of $G_{q}$ so that $a \notin B$. Then $n\left(a / b_{1}, b_{2}, \ldots, b_{n}\right) \geqslant k$ if and only if

$$
f=\sum_{\substack{x \in \mathbf{I} \\ x \notin\{a\} \cup B}}\{1+\eta(x-a)\} \prod_{i=1}^{n}\left\{1-\eta\left(x-b_{i}\right)\right\} \geqslant k 2^{n+1}
$$

To show that $f \geqslant k 2^{n+1}$, it is clearly sufficient to establish that $f>(k-1) 2^{n+1}$. Let

$$
g=\sum_{x \in \mathbf{F}_{q}}\{1+\eta(x-a)\} \prod_{i=1}^{n}\left\{1-\eta\left(x-b_{i}\right)\right\}
$$

From Lemma 3.3 we have

$$
g \geqslant q-\left\{(n-2) 2^{n}+2\right\} \sqrt{q}-\left\{2^{n}-2 n-1\right\}
$$

Now consider

$$
g-f=\sum_{x \in\{a\} \cup B}\{1+\eta(x-a)\} \prod_{i=1}^{n}\left\{1-\eta\left(x-b_{i}\right)\right\}
$$

If $g-f \neq 0$, then for some $y$ the product

$$
\{1+\eta(y-a)\} \prod_{i=1}^{n}\left\{1-\eta\left(y-b_{i}\right)\right\} \neq 0 \text {. }
$$

If $y=a$, then for (3.8) to hold we must have $\eta\left(a-b_{i}\right)=-1$ for all $i$. Hence, the term in (3.7) with $x=b_{i}$ contributes zero to the sum. Therefore, $g-f=2^{n}$, since each factor is 2 and one factor is 1 . If $y=b_{j}$ for some $j$, then for (3.8) to hold we must have $\eta\left(b_{j}-a\right)=1$. Hence, the term in (3.7) with $x=a$ contributes zero to the sum and the term with $x=b_{j}$ contributes $2^{n}$. Thus we conclude that $g-f \leqslant n 2^{n}$. So

$$
\begin{aligned}
f & \geqslant g-n 2^{n} \\
& \geqslant q-\left\{(n-2) 2^{n}+2\right\} \sqrt{q}-\left\{2^{n}-2 n-1\right\}-n 2^{n} \\
& =q-\left\{(n-2) 2^{n}+2\right\} \sqrt{q}-\left\{(n+1) 2^{n}-2 n-1\right\} .
\end{aligned}
$$

Now if inequality (3.6) holds, then $f>(k-1) 2^{n+1}$ as required. Since $a$ and $B$ are arbitrary, this completes the proof.

We have the following three corollaries to Theorem 3.1. 
Corollary 1 . Let $q \equiv 1(\bmod 4)$ be a prime power and $k$ a positive integer. Then $G_{q} \in \mathcal{G}(1,1, k)$ for every $q \geqslant 4 k+1$.

Proof: Inequality (3.6) shows that $q$ satisfies $q>4 k-3$. Since $q \equiv 1(\bmod 4)$, $q \geqslant 4 k+1$.

Corollary 2 . Let $q \equiv 1(\bmod 4)$ be a prime power and $k$ be a positive integer. If $q>(1+2 \sqrt{2 k})^{2}$, then $G_{q} \in \mathcal{G}(1,2, k) \cap \mathcal{G}(2,1, k)$.

Corollary 3 . Let $q \equiv 1(\bmod 4)$ be a prime number and $k$ be a positive integer. If $q>(5+\sqrt{16 k+34})^{2}$, then $G_{q} \in \mathcal{G}(1,3, k) \cap \mathcal{G}(3,1, k)$.

Remark 1. From Lemma 2.1 and Corollary 1 it follows that Theorem 3.1 is best possible for $n=1$.

REMARK 2. We have verified, by computer, that if $q \equiv 1(\bmod 4)$ is a prime power less than or equal to 1009 and $k$ is a positive integer with $q<(1+2 \sqrt{2 k})^{2}$, then $G_{q} \notin \mathcal{G}(1,2, k)$. We conjecture that this is true for all $q$. We can choose $a, b_{1}$ and $b_{2}$ in the proof of Theorem 3.1 so that $g-f=8$ and hence, by (3.5)

$$
f=g-8 \leqslant q+2 \sqrt{q}+1-8 .
$$

Consequently, $f<8 k$ for $q<(-1+2 \sqrt{2(k+1)})^{2}$. So the problem is to look at $(-1+2 \sqrt{2(k+1)})^{2} \leqslant q \leqslant(1+2 \sqrt{2 k})^{2}$.

\section{REFERENCES}

[1] W. Ananchuen and L. Caccetta, 'Graphs with a prescribed adjacency property', Austral. J. Combinatorics 6 (1992), 155-175.

[2] W. Ananchuen and L. Caccetta, 'On the adjacency properties of Paley graphs', Networks 23 (1993), 227-236.

[3] W. Ananchuen and L. Caccetta, 'On constructing graphs with a prescribed adjacency property', Austral. J. Combinatorics (to appear).

[4] A. Blass, G. Exoo and F. Harary, 'Paley graphs satisfy all first-order adjacency axioms', J. Graph Theory 5 (1981), 435-439.

[5] A. Blass and F. Harary, 'Properties of almost all graphs and complexes', J. Graph Theory 3 (1979), 225-240.

[6] B. Bollobás, Random graphs (Academic Press, London, 1985).

[7] J.A. Bondy and U.S.R. Murty, Graph theory with applications (The MacMillan Press, London, 1976).

[8] P. Erdōs and L. Moser, 'An extremal problem in graph theory', J. Austral. Math. Soc. 11 (1970), 42-47. 
[9] G. Exoo, 'On an adjacency property of graphs', J. Graph Theory 5 (1981), 371-378.

[10] R. Lidl and H. Niederreiter, Finite fields (Addison-Wesley, London, 1983).

[11] W.M. Schmidt, Equations over finite fields, an elementary approach, Lecture Notes in Mathematics 536 (Springer-Verlag, Berlin, Heidelberg, New York, 1976).

School of Mathematics and Statistics

Curtin University of Technology

GPO Box U1987

Perth WA 6001

Australia 